\title{
Pseudocoloring for a dual-phase grating method
}

\author{
Hai Tao Cai, MEMBER SPIE \\ Peter Chin Wan Fung \\ University of Hong Kong \\ Department of Physics \\ Pokfulam Road \\ Hong Kong
}

\begin{abstract}
A simple method for adjusting pseudocolor distributions of photographic images is described and demonstrated experimentally. This method is based on the technique for photographic phase image pseudocolor modulated by a grating. A reflecting ray path is introduced into a $4 f$ system to produce diffraction of the dual-phase grating so that the pseudocolor at the output plane can be adjusted. The merit of this method is that many pseudocolor images with different color distributions can be obtained for each diffraction order. () 1996 Society of Photo-Optical Instrumentation Engineers.
\end{abstract}

Subject terms: pseudocoloring encoding; phase image; chromaticity; CIE 1931 chromaticity diagram.

Paper 09115 received Nov. 6, 1995; accepted for publication Feb. 17, 1996.

\section{Introduction}

In general, the optical images obtained in various scientific applications are gray-level, black-and-white images. However, human observers perceive more complex color variations. Pseudocoloring of an image is a technique of introducing false colors to a monochrome image so that certain features of the image are easier to perceive. There are already many techniques for pseudocoloring of photographic images. ${ }^{1-6}$ A problem in the previous pseudocoloring techniques, however, is that sometimes the different densities of the image at the object plane may correspond to just one certain color at the output plane, thus leading to the wellknown "mixing color phenomenon."

To resolve such an ambiguity, we describe here a new method to adjust the color distribution at the output plane of the $4 f$ system. The key to this method is introducing a reflecting ray path into the $4 f$ system. By changing the position of a mirror in the reflecting ray path, we can obtain a series of color distributions for every diffraction order. Such color distributions would give us enough information to resolve the mixing color phenomenon.

In Sec. 2, we represent the mathematical formation of the problem and derive the relative light intensity distribution formulas at the output plane of the $4 f$ system for the zeroth-order spectrum and the positive first-order frequency spectrum. We then demonstrate the theory by experiments in Sec. 3 and present the chromaticity analysis for this method. Section 4 concludes the paper.

\section{Theory for Adjusting Pseudocolor Distribution in This Method}

The ray path diagram for adjusting color distribution of pseudocolor encoding images is shown in Fig. 1. A mirror and a semireflecting mirror were used as a set of white light input devices. A semireflecting mirror $N$ was inserted between an object plane $G$ and lens $L_{1}$ in the $4 f$ system while a reflecting mirror $M$ was placed at the left side of the $G$. The distance between $M$ and $G$ is $l$. In our experiments, a phase image encoded by grating was set at the $G$. A beam of collimating white light from a xenon arc lamp was re- flected by $N$ and illuminates a phase image encoded on $G$, and it was further reflected back by mirror $M$ to illuminate the phase image again. Having passed through the phase image, this beam of light was Fourier transformed by the first lens $L_{1}$ and was filtered at the frequency spectrum plane $S$ by a single-slit optical filter. It was then inverse Fourier transformed again by the second lens $L_{2}$ to form a pseudocolor image at an output plane $P$. According to the derivation represented later, the relative light intensity distribution at the output plane $P$ was found to be related to the distance $l$ from the phase image to mirror $M$. With such a setup, the relative light intensity distribution at the output plane can be changed with the distance $l$. Thereby, the color distributions of image at the output plane can be adjusted.

Now, suppose the coordinate at the object plane $G$ is $x$, at the frequency spectrum plane $S$ is $u$, and at the output $P$ is $x^{\prime}$. A phase image encoded by the grating is as shown as in Fig. 2. The amplitude transmittance described by Guo and $\mathrm{Chen}^{2}$ is

$$
\begin{aligned}
t(x)= & \exp \left(j \varphi_{2}\right)\left\{\operatorname{rect}\left(\frac{x}{d}\right)-\operatorname{rect}\left(\frac{x}{a}\right)[1-\exp (j \Delta \varphi)]\right\} \\
& * \sum_{-\infty}^{\infty} \delta(x-k d) \\
= & \exp \left(j \varphi_{2}\right)\left\{\sum_{-\infty}^{\infty} c_{k} \exp (j 2 \pi k x / d)-[1-\exp (j \Delta \varphi)]\right. \\
& \left.\times \sum_{-\infty}^{\infty} c_{k}^{\prime} \exp (j 2 \pi k x / d)\right\},
\end{aligned}
$$

where

$c_{k}^{\prime}=\frac{a}{d} \sin c\left(\frac{a k}{d}\right), \quad c_{k}=\sin c(k)$, 


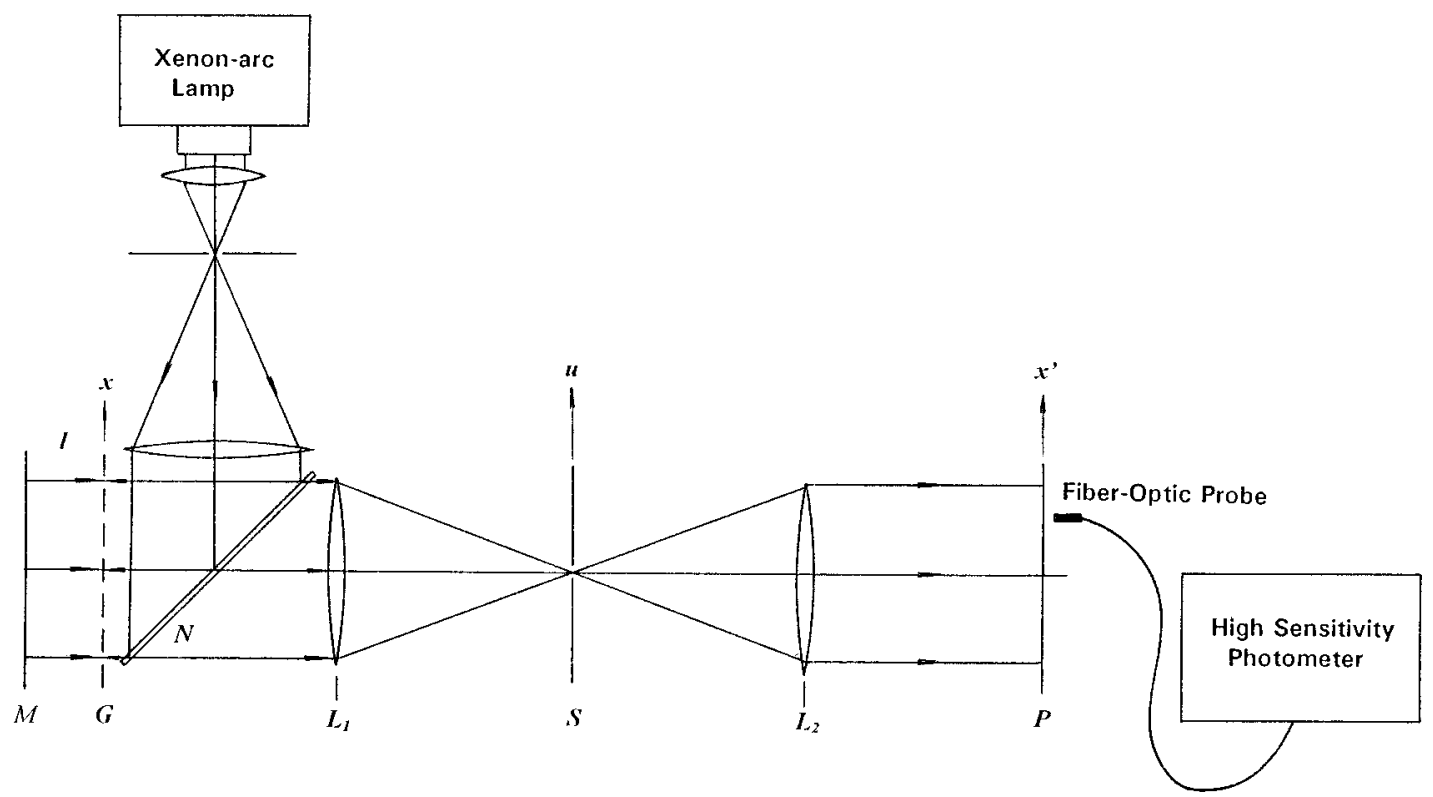

Fig. 1 Experimental device diagram for adjusting pseudocolor distributions of output images; $M$, reflecting mirror; $G$, object plane; $N$, beamsplitter $(1: 1) ; L_{1}$ and $L_{2}$, a pair of Fourier lenses; $S$, frequency spectrum plane; $P$, output plane; and $I$, distance between $M$ and $G$.

where $d$ is the grating constant; $\Delta \varphi(=2 \pi \Delta / \lambda)$ is the phase difference of the phase image encoded; and $\Delta$ is the optical path-length difference, which is related to the density of the input image. Such an aspect was discussed by Guo and Chen $^{2}$ and Zhang et al. ${ }^{6}$

A beam of collimating white light from the xenon arc lamp illuminates the phase image at the input plane $G$ and is reflected back to plane $G$ by mirror $M$ with a transmission distance of $2 l$. In terms of the well-known FresnelKirchhoff diffraction formula and Fourier transform theory, the complex amplitude distributions of the light intensity at the input plane $G$ and the frequency spectrums $S$ are $W_{1}(\xi)$ and $W_{2}(u)$, respectively. They are as follows:

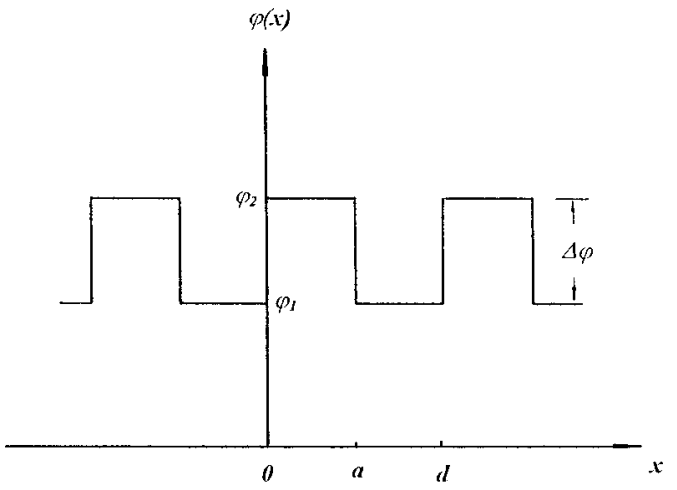

Fig. 2 Amplitude transmittance of a phase image encoded by a grating.

$$
\begin{aligned}
W_{1}(\xi)= & \frac{-j}{2 \lambda l} \exp (j 4 \pi l / \lambda) \int_{k=-\infty}^{\infty} t(x) \\
& \times \exp \left[j \frac{2 \pi}{\lambda} \frac{(x-\xi)^{2}}{4 l}\right] \mathrm{d} x
\end{aligned}
$$

and

$$
\begin{aligned}
W_{2}(u)= & \mathscr{F}\left[t(\xi) W_{1}(\xi)\right] \\
= & \frac{1-j}{2 \sqrt{l \lambda}} \exp (j 4 \pi l / \lambda) \exp \left(j 2 \varphi_{2}\right) \\
& \times \sum_{k=-\infty}^{\infty} \sum_{n=-\infty}^{\infty}\{\sin c(k)-[1-\exp (j \Delta \varphi)] \\
& \left.\times \frac{a}{d} \sin c\left(\frac{a k}{d}\right)\right\}\left\{\sin c(n)-[1-\exp (j \Delta \varphi)] \frac{a}{d}\right. \\
& \left.\times \sin c\left(\frac{a n}{d}\right)\right\} \exp \left(-j 2 \pi l \lambda k^{2} / d^{2}\right) \delta\left(u-\frac{n}{d}-\frac{k}{d}\right) .
\end{aligned}
$$

From Eq. (3), we know there are many orders of frequency spectrum bands appearing at the frequency spectrum plane $S$. As is usually the case, if dispersion is neglected and the sampling frequency (that is, the frequency of modulation grating) is twice as much as the highest frequency of the object image, these frequency spectrum bands would not coincide with each other. These spectrum bands can be filtered by a single-slit optical filter at the frequency spectrum plane $S$. The frequency spectrum distribution $W_{2}(u)$ 
for all orders is specified by the parameters $n$ and $k$. The zeroth-order frequency spectrum is depicted by $n+k=0$, the positive first-order frequency spectrum is represented by $n+k=1$, the negative first-order spectrum is described by $n+k=-1$, etc.

Now we take the first approximation [that is, neglecting the contributions of $W_{2}(u)$ for the values of $n, k$ greater than 1]. In view of Eq. (3), we can obtain the frequency spectra of all orders under such an approximation. Here, we just write down the zeroth-order and the positive first-order frequency spectra next.

For the zeroth-order frequency spectrum, the relationship $n+k=0$ must be satisfied. It concludes three sets of integers: $n=0, k=0 ; n=1, k=-1$; and $n=-1, k=1$. Substituting these integers into Eq. (3) and performing an inverse Fourier transform, the complex-amplitude distribution $g^{0}\left(x^{\prime}\right)$ at the output plane $P$ can be obtained:

$$
\begin{aligned}
g^{0}\left(x^{\prime}\right) & =\mathscr{F}^{-1}\left[W_{2}^{0}(u)\right] \\
& =\frac{1-j}{2 \sqrt{l \lambda}} \exp (j 4 \pi l / \lambda) \exp \left(j 2 \varphi_{2}\right)(A+j B),
\end{aligned}
$$

where

$$
\begin{aligned}
A= & 1-\frac{a}{d}+\frac{a}{d} \cos (2 \Delta \varphi)+\frac{2}{\pi^{2}} \sin \left(\frac{a \pi}{d}\right) \cos \left(\frac{2 \pi l \lambda}{d^{2}}\right) \\
& -\frac{4}{\pi^{2}} \sin ^{2}\left(\frac{a \pi}{d}\right) \cos \left(\Delta \varphi-\frac{2 \pi l \lambda}{d^{2}}\right) \\
& +\frac{2}{\pi^{2}} \cos \left(2 \Delta \varphi-\frac{2 \pi l \lambda}{d^{2}}\right), \\
B= & \frac{a}{d} \sin (2 \Delta \varphi)-\frac{2}{\pi^{2}} \sin ^{2}\left(\frac{a \pi}{d}\right) \sin \left(\frac{2 \pi l \lambda}{d^{2}}\right) \\
& -\frac{4}{\pi^{2}} \sin \left(\frac{a \pi}{d}\right) \sin \left(\Delta \varphi-\frac{2 \pi l \lambda}{d^{2}}\right) \\
& +\frac{2}{\pi^{2}} \sin \left(2 \Delta \varphi-\frac{2 \pi l \lambda}{d^{2}}\right) .
\end{aligned}
$$

Thus, the relative light intensity distribution for the zerothorder frequency spectrum at the output plane $P$ is

$I^{0}\left(x^{\prime}\right)=\left|g^{0}\left(x^{\prime}\right)\right|^{2}=\frac{A^{2}+B^{2}}{2 l \lambda}$.

Similarly, we can get the relative light intensity distribution $I^{+1}\left(x^{\prime}\right)$ for the positive first order at the output plane $P^{\prime}$

$$
\begin{aligned}
I^{+1}\left(x^{\prime}\right)=\left|g^{+1}\left(x^{\prime}\right)\right|^{2}= & \frac{2}{\pi^{2} l \lambda} \sin ^{2}\left(\frac{\pi a}{d}\right) \cos ^{2}\left(\frac{\pi l \lambda}{d^{2}}\right) \\
& \times\left(A^{\prime 2}+B^{\prime 2}\right),
\end{aligned}
$$

where

$$
\begin{aligned}
& A^{\prime}=1-\frac{a}{d}+\left(\frac{2 a}{d}-1\right) \cos (\Delta \varphi)-\frac{a}{d} \cos (2 \Delta \varphi), \\
& B^{\prime}=\left(\frac{2 a}{d}-1\right) \sin (\Delta \varphi)-\frac{a}{d} \sin (2 \Delta \varphi) .
\end{aligned}
$$

In Eqs. (5) and (6), we choose the $d=\frac{1}{20} \mathrm{~mm}$ and $a$ $=d / 2$. When the $\Delta \varphi$ and $\lambda$ are the constants, the relative light intensity distributions are functions of the variable $l$. Figure 3 shows that the relative light intensity of the zerothorder output changes with $l$, where the $\Delta \varphi$ is $2 \pi / 3$ and the parameters $\lambda$ are set as 6300,5205 , and $4205 \AA$, respectively. From this figure we know that the relative light intensities for every wavelength have their cyclic variation. The longer the wavelength is, the shorter is the cycle. For different $l$ values, the intensity ratios of the three wavelengths are different; they can mix to form different colors, which is why the color distributions can be adjusted by changing $l$.

\section{Experimental Demonstration and Chromaticity Analysis}

\subsection{Experimental Demonstration}

In our experiments, a set of xenon arc lamps was used as the white light source. A grating set with $d=\frac{1}{20} \mathrm{~mm}$ was used to produce the phase image, employing the method discussed by Guo and Chen. ${ }^{2}$ We placed a uniform phase image ( $\Delta \varphi=$ constant) at the plane $G$ of Fig. 1, and placed, respectively, three color filters $(6300,5205$, and $4205 \AA)$ at the plane $S$ of Fig. 1. At the output plane $P$, we use a set of photometers to measure the relative light intensity. For a color filter (a fixed $\lambda$ value), we measured a range of values of $I^{0}\left(x^{\prime}\right)$ corresponding to a range of $l$ values. The process was repeated for three color filters with $\lambda$ specialized as before. The experimental results $I^{0}\left(x^{\prime}\right) \sim l$ with three $\lambda$ values are indicated in Fig. 4.

Comparing Fig. 4 with Fig. 3, we observe that the theoretical intensity distribution curves match the experimental ones rather well. The experimental curves in Fig. 4 have the same cycle variation as that in Fig. 3, and the cycle pertaining to the longer wavelength is also shorter than that of the shorter wavelength. We can say that the theoretical consequence is in agreement to experimental result.

\subsection{Chromaticity Analysis}

In terms of chromaticity theory, one color corresponds to a chromaticity coordinate on the CIE 1931 chromaticity diagram. The chromaticity coordinate is related to the CIE 1931 tristimulus values $X, Y$, and $Z$, which can be specified by following formulas ${ }^{7}$ :

$X=k \sum_{\lambda} I(\lambda) S(\lambda) \bar{x}(\lambda) \Delta \lambda$,

$Y=k \sum_{\lambda} I(\lambda) S(\lambda) \bar{y}(\lambda) \Delta \lambda$, 


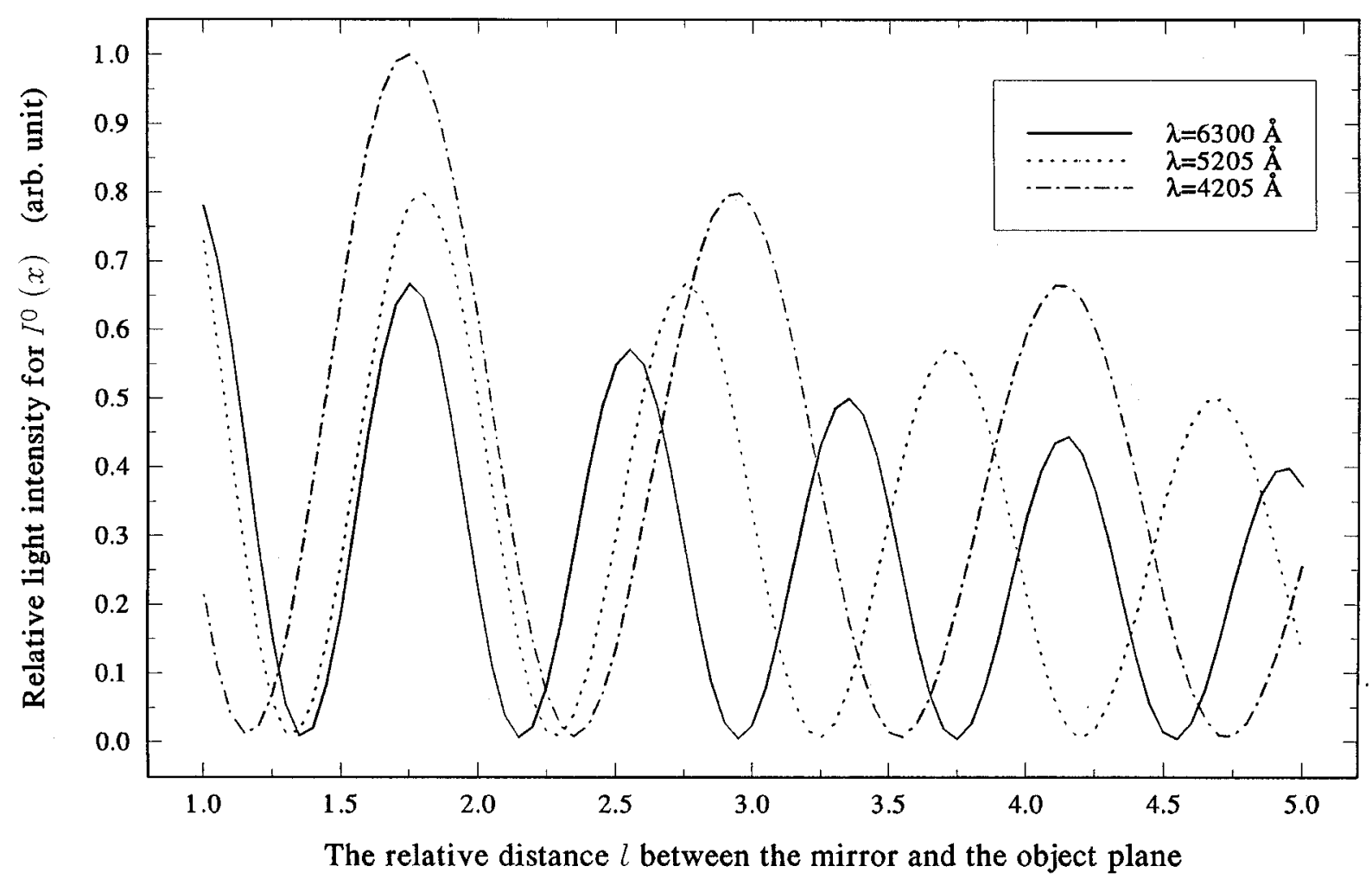

Fig. 3 Theoretical curves of the relative light intensity distribution of the three wavelengths 6300 , 5205 , and $4205 \AA$ for the zeroth-order output ( $\Delta \varphi$ is a certain constant).

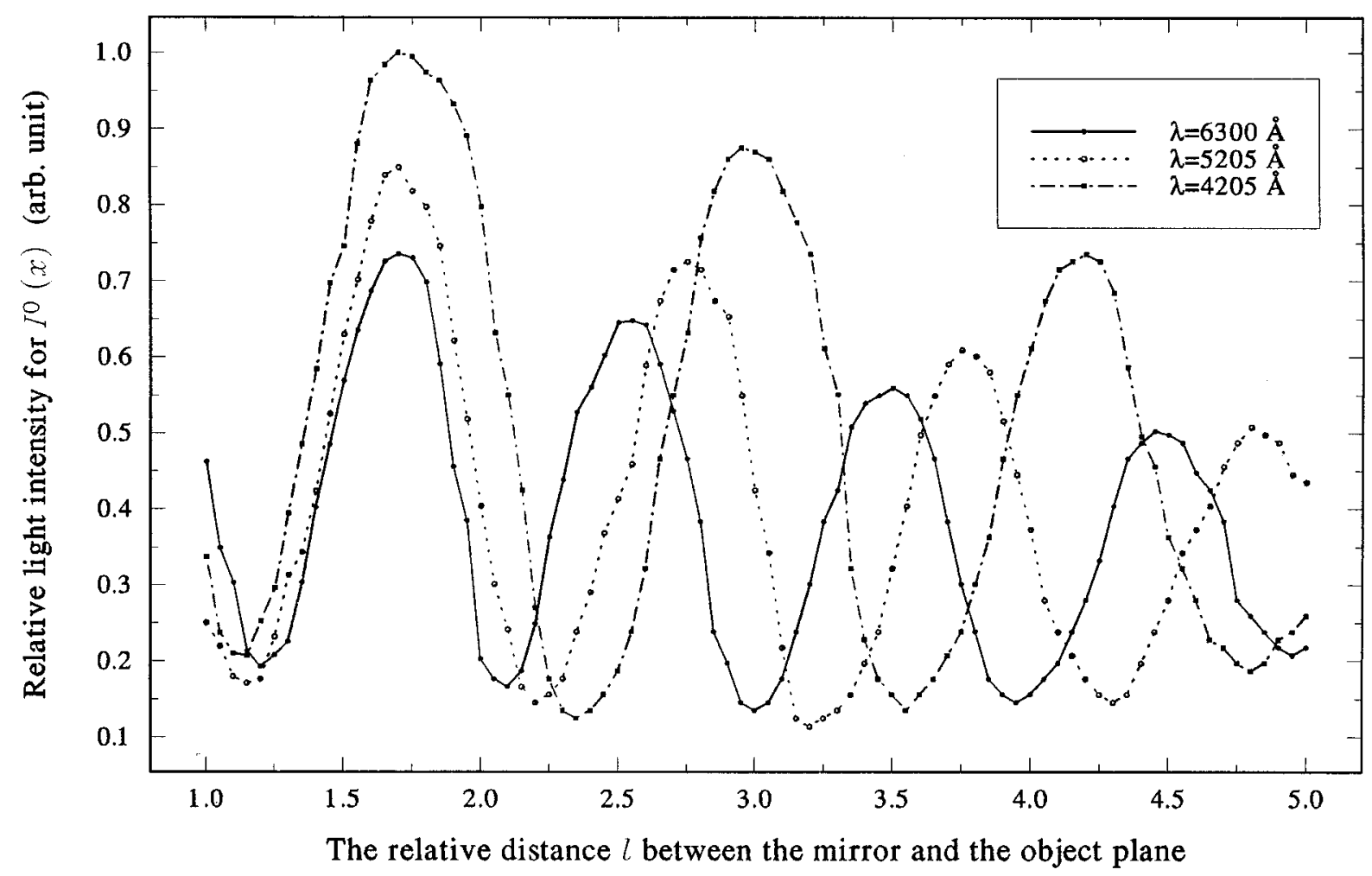

Fig. 4 Experimental curves of the relative light intensity distribution of the three wavelengths 6300 , 5205 , and $4205 \AA$ for the zeroth-order output $(\Delta \varphi$ is a certain constant). 


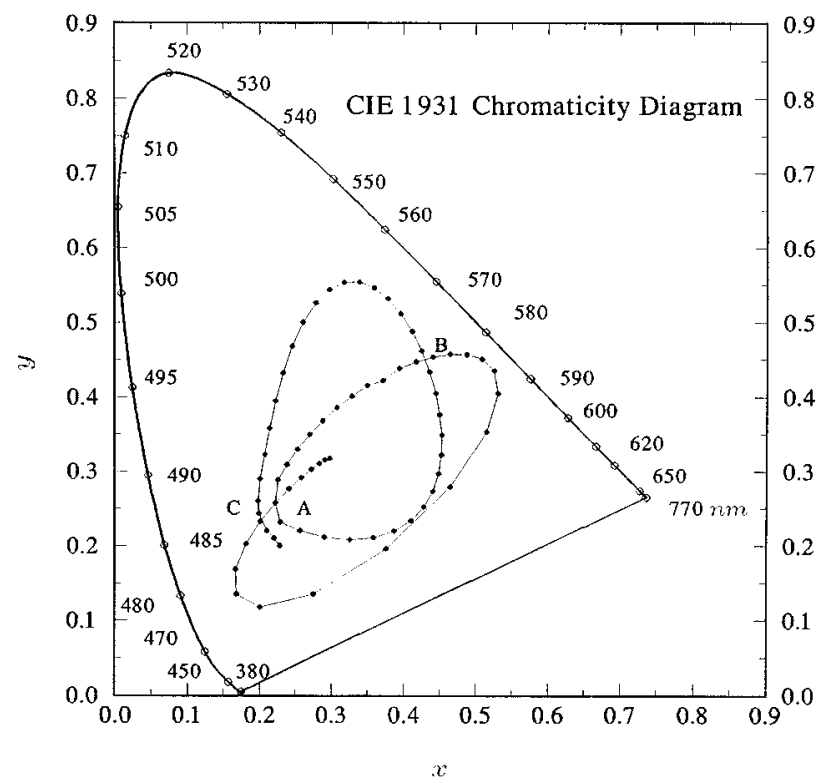

Fig. 5 For the zeroth-order output, chromaticity coordinate changes with phase difference $\Delta \varphi$ of input image while the distance / is considered as a constant. The cross points $A, B$, and $C$ show the mixing color phenomenon, where the different $\Delta \varphi$ correspond to the same chromaticity point.

$Z=k \sum_{\lambda} I(\lambda) S(\lambda) \bar{z}(\lambda) \Delta \lambda$

where $k$ is a normalizing factor; $I(\lambda)$ is the relative light intensity $I^{0}\left(x^{\prime}\right)$ or $I^{+1}\left(x^{\prime}\right)$ appearing in Eq. (5) or Eq. (6); and $S(\lambda)$ is the relative spectral power distribution of a xenon arc lamp, and can be substituted for by that of the CIE 1931 standard illuminate $D_{65}$. The quantities $\bar{x}(\lambda)$, $y^{-}(\lambda)$, and $\bar{z}(\lambda)$ are the color-matching functions of the CIE 1931 standard colorimetric observer tristimulus values. The data of $S(\lambda), \bar{x}(\lambda), \bar{y}(\lambda)$, and $\bar{z}(\lambda)$ have been tabled for the whole range of visual wavelengths, which can be obtained from Ref. 7. The chromaticity coordinates in the CIE 1931 chromaticity diagram can there be expressed as

$x=X /(X+Y+Z), \quad y=Y /(X+Y+Z)$.

Note that different densities of input image correspond to different $\Delta \varphi$, which in turn correspond to different colors on the output plane or corresponds to different chromaticity coordinate points in the CIE 9131 chromaticity diagram.

In the previous pseudocoloring methods,${ }^{1-6}$ the chromaticity coordinates $(x, y)$ are only functions of the phase difference $\Delta \varphi$. The relationship curve between the chromaticity coordinates $(x, y)$ and $\Delta \varphi$ is shown ${ }^{3}$ in Fig. 5. Every chromaticity coordinate point at the curve in Fig. 5 represents a kind of color. We observe from Fig. 5 that the curve has cross points $A, B$, and $C$. These cross points mean that two different values of $\Delta \varphi$ correspond to just one chromaticity coordinate. In other words, two different densities of

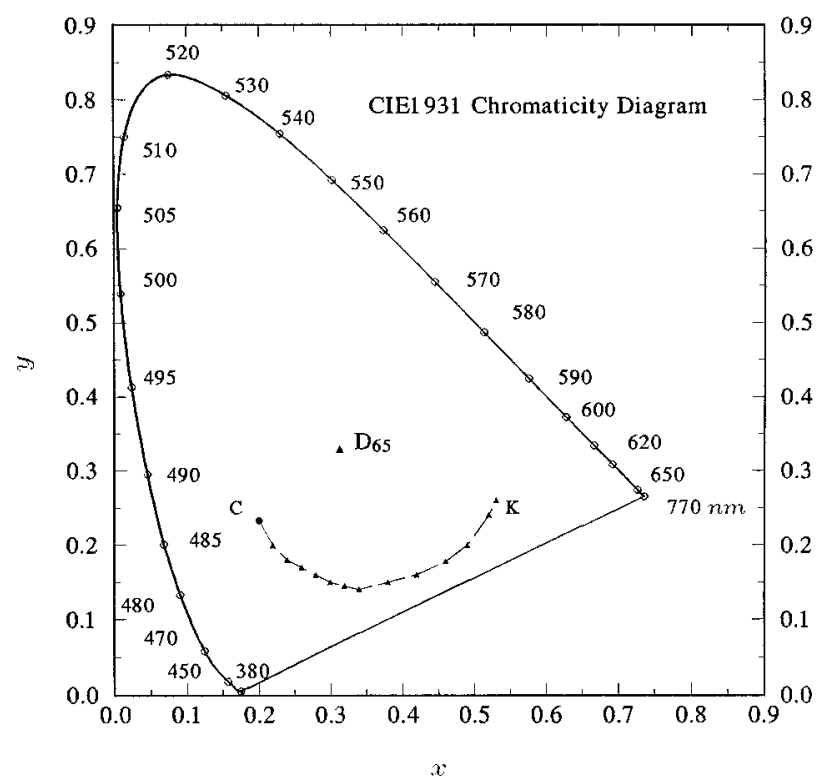

Fig. 6 For the zeroth-order output and a certain $\Delta \varphi$ value, one chromaticity point $C$ can be extended to a curve $C K$ by changing the distance $/$ between $M$ and $G$ in Fig. 1.

the input image correspond to the same color. This is socalled the mixing color phenomenon, which is a major drawback.

In our method, the chromaticity coordinates $(x, y)$ are not only functions of $\Delta \varphi$ but also functions of the length variable $l$, which is introduced by our method. We can change the length value $l$ to expand a cross point (just mentioned) into a curve, which means we can transfer one kind of color (for one chromaticity coordinate) to several colors (for the curve) so that the color range can be expanded and the mixing color phenomenon can be avoided.

Since a cross point has two different $\Delta \varphi$ values, for a certain cross point, we assume one of the $\Delta \varphi$ values is a constant and take $l$ as a variable. Substituting Eq. (5) into Eqs. (7) and (8), and summing the whole range of visual wavelengths for every $l$ value, we obtain the values of $X$, $Y$, and $Z$. The chromaticity coordinates $(x, y)$ can then be calculated from Eq. (8). Different $l$ values correspond to different coordinates $(x, y)$. Plotting these $(x, y)$ coordinates in the CIE 1931 chromaticity diagram, we can obtain a chromaticity curve $C K$, which is shown in Fig. 6. From this curve, we can select different colors on the $C K$ curve rather than only one color. As the color saturation of the edge of the CIE 1931 chromaticity is better than of the center, we see that the color saturation of the output image can be improved using our method.

\section{Conclusions}

In our experiments, we only need to add a plane mirror and a semireflecting mirror in the well-known $4 f$ system. We can simply adjust the positions of the mirror to obtain the images of different color distributions at the output plane.

Figure 1 is our experimental setup. For the zeroth-order output, using $\lambda_{1}=6300 \AA, \lambda_{2}=5205 \AA$, and $\lambda_{3}=4205 \AA$ as 
the wavelength parameters, the theoretical intensity distribution curves and the corresponding experimental curves are presented in Figs. 3 and 4, respectively. The two sets match rather well.

The results of chromaticity analysis are indicated in Figs. 5 and 6 . We observe that one chromaticity point can be expanded into one curve. Hence, the mixing color phenomenon can be avoided and the color saturation can be improved using the method introduced here.

\section{References}

1. T. H. Chao, S. L. Zhuang, and F. T. S. Yu, "White-light pseudocolor density encoding through contrast reversal," Opt. Lett. 5(6), 230-232 (1980).

2. L. R. Guo and Z. P. Chen, "Pseudocolor density encoding through phase modulation," Acta Opt. Sinica 4(2), 145-147 (1984).

3. H. Kang, "Characteristics of phase encoding plates formed by beaching silver-halide films,' Acta Opt. Sinica 5(8), 750-753 (1984).

4. H. M. Liu, "Black-white image density pseudo-color encoding achieved by Mach-Zehnder interferometer," Laser J. 9(1), 44-46 (1986) (in Chinese).

5. J. F. Zhang and M. W. Yu, "A pseudo-color encoder using photodichroism in silver chloride emulsion," Acta Opt. Sinica 1(2), 145152 (1982).

6. J. J. Zhang, S. Y. Wang, D. H. Liu, and F. L. Peng, "The pseudocoloring of an encoded phase-picture," Acta Opt. Sinica 5(10), 944-950 (1985).

7. D. B. Judd and Gunter Wyszecki, Color in Business, Science and Industry, Chap. 2, pp. 144-188, John Wiley \& Sons, New York (1975).

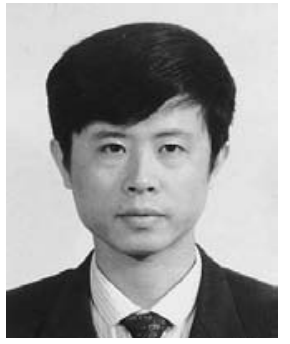

Hai Tao Cai received his BSc degree in physics from Sichuan University, China, in 1982, took up a teaching post, and completed his MSc degree in physics in 1988 also at Sichuan University. He enrolled as a $\mathrm{PhD}$ student in the University of Hong Kong in 1994 under the supervision of Prof. P. C. W. Fung, working on photoacoustics techniques and color optics.

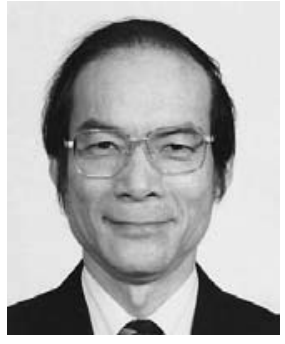

Peter Chin Wan Fung received his university education in Australia. He completed his BSc in physics, a BSc with honors in radio astronomy, and $\mathrm{AhD}$ in radio astronomy in the 1960s and became a research associate at the University of Montreal, Canada, in 1967 to 1968 . He was at Stanford University as research associate, research physicist, and scientific consultant from 1968 to 1970 , and part of 1971 . He has been with the University of Hong Kong since 1970 and has been a visiting professor during some study leaves. Currently he is a professor of physics and directs the Centre of Materials Science at the University of Hong Kong. Prof. Fung's interests include pure and applied physics, including various aspects of optics, superconductivity, superfluidity, plasma physics, soliton physics, and astrophysics. He has published well over 200 research articles in international journals. 